\title{
Evolution of uranium dislocation structure at different-rate deformation and different-temperature annealing
}

\author{
A.E. Shestakov and I.V. Artamonov \\ Russian Federal Nuclear Center - Zababakhin All-Russia Research Institute of Technical Physuics, \\ Snezhinsk, Russia
}

\begin{abstract}
The Williamson-Hall method based on analyzing the angular dependence of diffraction maximum broadening is used to study the dislocation structure. Densities of chaotically arranged dislocations on samples after their different-rate deformation are measured. Consideration is given to the dislocation structure of a spherical uranium sample after its shock-wave loading, as well as to the dislocation structure of model samples deformed at the rate of $\sim 10^{-3} \mathrm{~s}^{-1}$ up to $5 \%, 20 \%$, and $60 \%$ relative deformation. After deformation, samples were vacuum annealed at 200C, 400C, 600C, 720C, and 850C (in alpha-, beta-, and gamma-phases). Samples were cooled at the rate of $\sim 10^{\circ} / \mathrm{min}$. Results for samples subjected to different-rate deformation and different-temperature annealing are compared. In the dislocation structure of the sample recovered after shock-wave loading, special features that cannot be described with the help of mechanisms observed at the low-rate deformation and cooling were elucidated. Systematic investigations of the uranium dislocation structure at the increasing speed of loading are to be continued).
\end{abstract}

